\title{
Zonas de fondeo, interferencias con actividades pesqueras uruguayas, y elementos para la planificación espacial marina en el Río de la Plata
}

Yamandú H. Marín

Ciencia y Tecnología Marina, Facultad de Ciencias, Universidad de la República, Uruguay

\section{Guzmán López}

Dirección Nacional Recursos Acuáticos, Ministerio de Ganadería, Agricultura y Pesca (MGAP), Uruguay

\section{Julio F. Chocca}

Dirección Nacional Recursos Acuáticos, Ministerio de Ganadería, Agricultura y Pesca (MGAP), Uruguay

\author{
Mónica Gómez \\ Ciencia y Tecnología Marina, Facultad de Ciencias, Universidad de la República, Uruguay
}

Recibido: 5 de marzo de 2018. Aceptado: 21 de mayo de 2018.

\section{Resumen}

Las zonas de alijo y transferencia de carga fueron definidas en el Tratado del Río de la Plata en 1973, ubicando dos a cada lado del río. Con el incremento de la actividad económica regional también crecieron el número y tamaño de los buques e indujo al trazado de nuevas zonas de fondeo y espera aumentando de $236 \mathrm{~km}^{2}$ en 1977 a $600 \mathrm{~km}^{2}$ en 2014. Dado que estas zonas se ubican en áreas de pesca tradicionales de la flota pesquera uruguaya se evaluó el número de buques, tipo y destino de aquellos fondeados en las zonas de alijo y espera, durante 6 meses entre 2017 y 2018 en base a datos provistos por el Sistema de Identificación Automático (AIS, Automatic Identification System). Se encontró que el número de buques fondeados en estas zonas osciló en 33 cada día, que hay un ingreso y egreso continuo, y que el tiempo de residencia de cada buque fue de 3,6 días con una gran dispersión de valores. El tipo de buque más común fue el transporte a granel, y los destinos más frecuentes fueron San Lorenzo y Rosario en Argentina. La mayor actividad en la zona más profunda del río próxima a la costa uruguaya donde se realiza pesca costera contrasta con la escasa o nula ocupación de las zonas de alijo próximas a la costa argentina. Se discuten los alcances de esta asimetría y se sugiere una revisión de la asignación de los espacios en Río de la Plata en el marco del Tratado.

\section{Palabras clave}

Zonas de fondeo Río de la Plata Interferencias Pesquerías Planificación espacial marina 


\section{Anchorage areas, interference with Uruguayan fishing activities, and elements for marine spatial planning in the Río de la Plata}

\begin{abstract}
Lightering and cargo transfer areas were defined in the Treaty of Río de la Plata in 1973, placing two on each side of the river. The increase of regional activity also increased the flow of goods, services, and fluvial and maritime transport. The growing number and size of transport vessels has led to the establishment of new anchorage zones rising from $236 \mathrm{~km}^{2}$ in 1977 to $600 \mathrm{~km}^{2}$ in 2014. Given that these zones are located in traditional fishing areas of the Uruguayan fishing fleet, the number of vessels, type and destination of vessels anchored in the various lightering and awaiting zones was evaluated along 6 months between 2017 and 2018, based on the data provided by the Automatic Identification System (AIS). It was found that the number of vessels anchored in these zones ranged 33 vessels each day, that there was a continuous vessels entry and exit, and that the residence time of each vessel was 3.6 days with a large dispersion of values. The most common type of ship was the Bulk Carrier, and the most frequent destinations were San Lorenzo and Rosario in Argentina. The higher activity in the deepest part of the river near the Uruguayan coast, where coastal fishing is carried out, contrasts with the scarce or null occupation of the lightering and cargo transfer areas near the coast of Argentina. The scope of this asymmetry is discussed and a revision of the space allocation in Rio de la Plata is suggested under the Treaty framework.
\end{abstract}

\section{Introducción}

\section{El Río de la Plata}

El Río de la Plata (RDP) está ubicado en el margen occidental de América del Sur entre las latitudes $34^{\circ} 00^{\prime} \mathrm{S}$ y $36^{\circ} 30^{\prime} \mathrm{S}$, y $055^{\circ} 00^{\prime} \mathrm{W}$ y $058^{\circ} 30^{\prime} \mathrm{W}$ de longitud. Es un cuerpo de agua cuya superficie se estima en $30.000 \mathrm{~km}^{2}$, con una longitud de $280 \mathrm{~km}$ a lo largo del eje, una sección transversal que aumenta hacia el sureste desde $2 \mathrm{~km}$ en Punta Gorda, hasta $230 \mathrm{~km}$ entre Punta del Este y Punta Rasa, y una profundidad media de $10 \mathrm{~m}$ (CARP, 2017; Fossati et al., 2014). Allí converge la Cuenca del Plata, una de las mayores del continente y que comparten cinco países (Brasil, Argentina, Paraguay, Bolivia y Uruguay). Los ríos Paraná, Uruguay, y una densa red de tributarios proveen de agua dulce y servicios ecosistémicos a una extensión mayor a 3 millones de $\mathrm{km}^{2}$, con una población que supera los 100 millones de personas (CIC, 2012), y donde se estima que se genera el $70 \%$ del Producto Bruto Interno (PBI) de los cinco países.

El RDP se encuentra en la interfaz entre el drenaje de la cuenca y el Océano Atlántico, constituye el límite geográfico entre Argentina y Uruguay, y es una de las principales vías de entrada y salida de productos de la región.

La demarcación de los límites entre ambos países fue un proceso que insumió décadas de conflictos y acuerdos desde el siglo XIX, y que fue resuelto con el Tratado del Río de la Plata y su Frente Marítimo en 1973. El tratado estableció un régimen aguas de uso común, zonas de jurisdicción exclusiva de cada país, dispuso normas sobre la libertad de navegación, canales e infraestructura, una zona común de pesca más allá del mar territorial, y un sistema administrativo binacional asentado en comisiones administrativas, científicas y técnicas: Comisión Administradora del Río de la Plata, (CARP, 2017) y Comisión Técnico Mixta del Frente Marítimo (CTMFM, 2017).

\section{Keywords}

Anchorage areas La Plata River Interferences Fisheries

Marine spatial planning

\section{Palavras-chave}

Áreas de fundeio Rio da Prata Interferências Pescarias Planejamento espacial marinho 
Entre los aspectos que involucra, se incluyen secciones relativas al uso y mantenimiento de los canales de navegación; obras e infraestructura; operaciones y zonas de alijo y complemento de carga; y recursos del suelo y subsuelo.

El capítulo IV del Tratado se refiere a las "zonas de alijo, complemento y transferencia de carga”, áreas destinadas a aligerar o aumentar la carga de una embarcación, y por lo tanto su calado, de acuerdo a la profundidad de su próximo puerto de destino. Corresponden dos de estas zonas a cada país, situadas a ambos lados del RDP (zonas "A", "B", "C", y "D"), en "... número igual de zonas situadas en la proximidad de las costas de cada Parte, pero fuera de las respectivas zonas costeras" (art. 28). Entre su delimitación en 1973 y 2014 estas zonas se fueron modificando: el área "A" fue reubicada en el año 2000 (zona "Alfa"); las áreas "B" o "Bravo", "C" o "Charlie", y "D" o "Delta" fueron demarcadas en 1977; la de "Servicios" en el año 2000, y en 2014 se incorporaron dos nuevas zonas (zonas de espera "Este" y "Oeste") aumentando la superficie total de 21,4 $\mathrm{mn}^{2}$ a $175,1 \mathrm{mn}^{2}$ (millas náuticas cuadradas) en 42 años (Figura 1, Cuadro 1).

La zona STS (ship to ship) fue originalmente un polígono donde los buques transferían cargas líquidas de uno a otro, ambos en movimiento. Posteriormente se designó una serie de puntos entre los cuales se realiza la operación además de puntos de espera.

Cuadro 1. Evolución de las áreas de alijo, complemento de carga, y espera y fondeo en el Río de la Plata y O. Atlántico. Superficie en $\mathrm{mn}^{2}$ (millas náuticas cuadradas, $1 \mathrm{mn}^{2}=3,43 \mathrm{~km}^{2}$ ), profundidad media (z, en $\mathrm{m}$ ) y período de vigencia. El área Alfa fue modificada en el año 2000. *DISMAR (2017). Disposición Marítima, Armada Nacional, Uruguay. "* Restricción en un radio de 1 mn alejados de los buques en operaciones STS (ship-to-ship) sin superficie definida. Fuente: elaborado en base a la revisión de la normativa (DISMAR, 2017).

\begin{tabular}{|l|c|c|c|c|c|l|}
\hline Zona & $\mathbf{1 9 7 3}$ & $\mathbf{1 9 7 7}$ & $\mathbf{2 0 0 0}$ & $\mathbf{2 0 1 4}$ & $\mathbf{z ~ ( m )}$ & Referencia \\
\hline A 1973 & 6,7 & 6,7 & & & 9,5 & $\begin{array}{l}\text { Tratado Río de la Plata; } \\
\text { DISMAR* 9, 1977 }\end{array}$ \\
\hline Bravo & 14,7 & 14,7 & 14,7 & 14,7 & 13,5 & DISMAR* 9, 1977 \\
\hline Charlie & & 39,2 & 39,2 & 39,2 & 16,5 & $\begin{array}{l}\text { Tratado Río de la Plata; } \\
\text { DISMAR 9, 1977 }\end{array}$ \\
\hline Delta & & 8,3 & 8,3 & 8,3 & 24,0 & DISMAR 9, noviembre 1977 \\
\hline Alfa 2000 & & & 20,1 & 20,1 & 14,8 & DISMAR 79, febrero 2000 \\
\hline Servicios & & & 7,8 & 7,8 & 10,4 & DISMAR 79, febrero 2000 \\
\hline Este & & & & 35,1 & 21,9 & DISMAR 156, enero 2015 \\
\hline Oeste & & & & 50,4 & 13,2 & DISMAR 156, enero 2015 \\
\hline Total mn2 & 21,4 & 68,9 & 90,0 & 175,1 & & \\
\hline STS & & & & $* *$ & & DISMAR 167, junio 2017 \\
\hline
\end{tabular}

La Figura 1 muestra las zonas de fondeo y alijos en el Río de la Plata definidas por el Tratado del Río de la Plata (1973) y modificaciones posteriores en los años 1977, 2000 y 2015. Se indica la zona de espera de practicaje en La Plata que se encuentra en la franja de jurisdicción exclusiva de Argentina y no fue incluida en este trabajo.

\section{Interacciones con pesquerías}

Entre las múltiples actividades que se realizan en las aguas comunes del RDP se destacan el transporte comercial y la pesca debido al número de embarcaciones que participan. Uruguay cuenta con una flota pesquera compuesta por 518 embarcaciones artesanales (menores de 10 TRB, Toneladas de Registro Bruto), el 68\% de las cuales operan en el RDP y O. Atlántico a distancias menores a 15 millas de la costa con redes de enmalle y anzuelos. La flota industrial (mayores a $10 \mathrm{TRB}$ ) está compuesta por 62 buques 


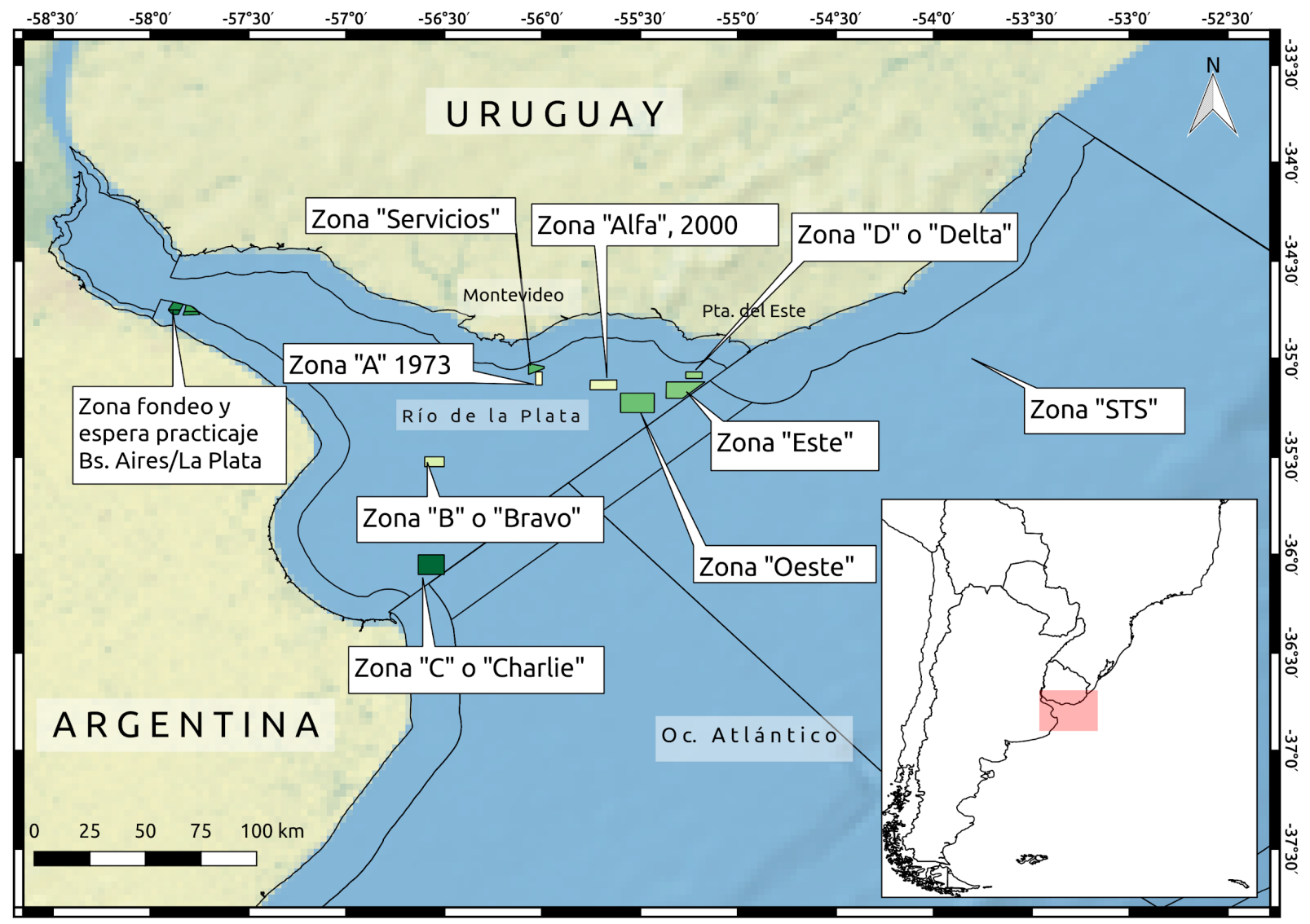

clasificada en 4 categorías (Decreto $\left.\mathrm{N}^{\circ} 149 / 997\right)$ : 24 buques de $45 \mathrm{~m}$ de eslora en la categoría "A" que capturan merluza (Merluccius hubbsi) en la plataforma continental; 33 buques costeros en la categoría "B" de $23 \mathrm{~m}$ de eslora que capturan corvina (Micropogonias furnieri) y pescadilla (Cynoscion guatucupa) en el RDP; 4 barcos en la categoría "C" dirigidos a especies y zonas diversas; y 1 buque en la categoría " $\mathrm{D}$ " que opera en aguas internacionales. Las categorías "A" y "B" forman el conjunto mayor (92\% de la flota), y operan con redes de arrastre de fondo en pareja o con portones, mientras que las categorías "C" y "D" son más heterogéneas en dimensiones, artes de pesca, especies objetivo y zonas de trabajo (DINARA 2015; DIEA 2017).

Diferentes agrupaciones del sector pesquero (armadores, tripulantes) han indicado que las zonas de espera y fondeo se han convertido en un problema debido a que ocupan un gran espacio de zonas tradicionales de pesca, generan ruido, alteran el fondo, remueven sedimentos y acumulan desperdicios, afectando especialmente a la flota de la categoría "B” (Cámara de Representantes, 2015; Observador, 2015; Riguetti, 2016). El área de operación de este segmento de flota industrial abarca el RDP desde el oeste de Montevideo hasta el límite lateral con Brasil en el O. Atlántico, hasta profundidades de $50 \mathrm{~m}$. Las operaciones varían estacionalmente, se ha cartografiado el esfuerzo de pesca en la zona utilizando emisiones de VMS, vessel monitoring system (Chocca et al. 2007; Chocca et al., 2016; Marín et al. 2007, 2013) y existen registros de actividad al menos desde 1940 (Barattini, 1943)(Figura 2).

Tanto para el transporte como para la pesca, en ambos casos existen reglamentaciones excluyentes (ej. no se permiten operaciones de pesca en canales de navegación), y propias (ej. áreas de veda de corvina en el oeste de Montevideo, o prohibición de uso de red de arrastre en una franja de 5 millas desde la costa). En algunos
Figura 1. Zonas de fondeo y alijos en el Río de la Plata definidas por el Tratado del Río de la Plata (1973) y modificaciones posteriores en los años 1977 , 2000 y 2015 . Fuente: elaborado en base a la revisión bibliográfica. 


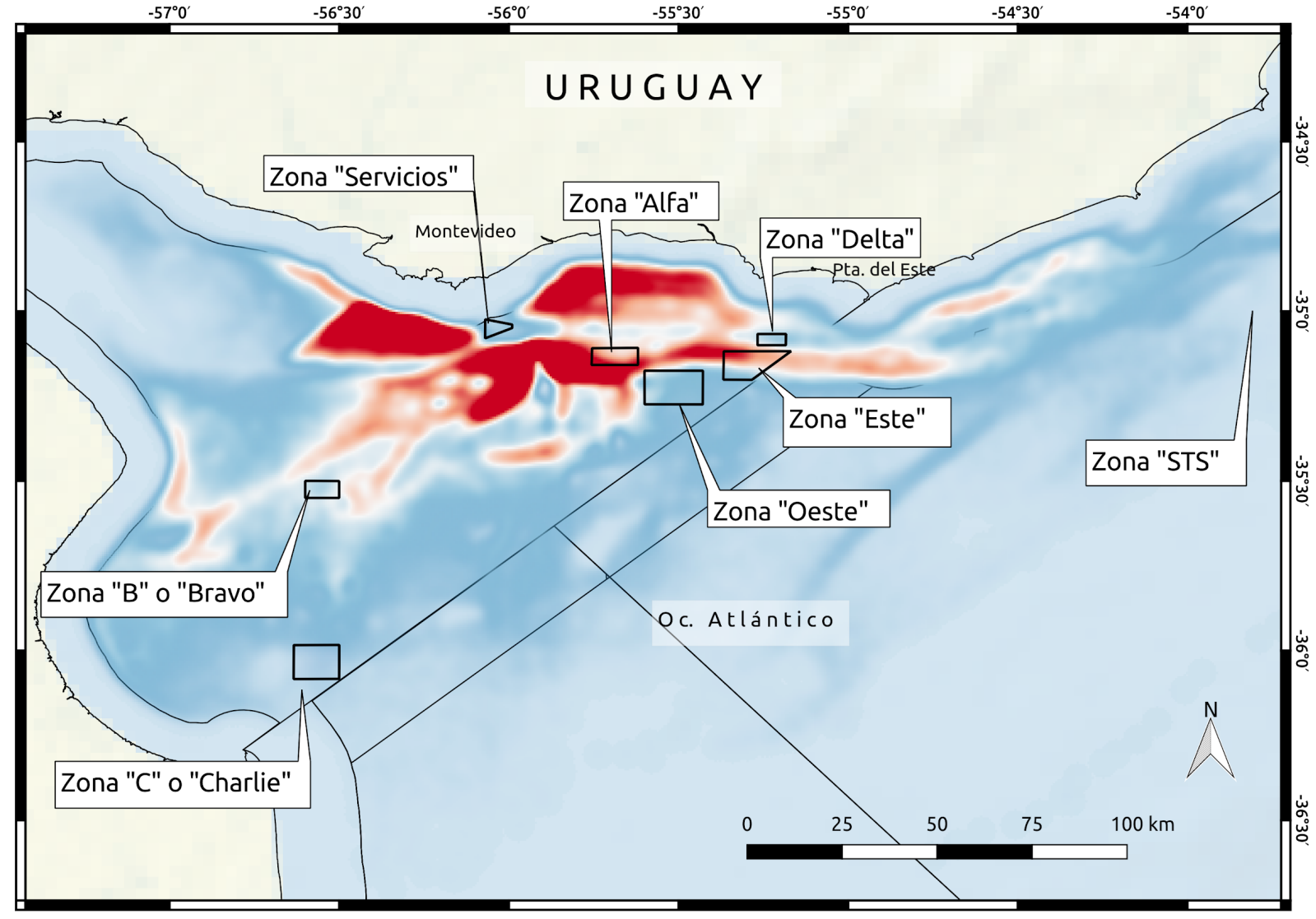

casos la exclusión es de hecho, ya que las unidades de pesca evitan utilizar redes de arrastre cerca de anclas o cadenas de buques fondeados aunque no haya una reglamentación específica.

La extensión de la superposición e interacciones entre buques de transporte y de pesca está caracterizada en forma general (Marín et al. 2013), no cuantificada, y existen diferentes versiones del número de barcos involucrados. En este sentido, el objetivo del trabajo es describir la actividad en las zonas de fondeo, alijo, espera o transferencia de carga que se superponen con las operaciones pesqueras de la flota industrial uruguaya. En particular qué áreas se utilizan, cuál es su ocupación y las características de los buques que lo hacen.

\section{Material y métodos}

Se utilizó la denominación genérica de "zona de fondeo" a todas aquellas señaladas en el Cuadro 1, ya sea de "alijo", de "espera y fondeo", o de cualquier naturaleza donde fueran observados buques fondeados en las aguas de uso común del RDP (excluyendo franjas adyacentes a la costa de cada país). Se consideró "fondeado" a todo buque que permaneció en la misma posición durante más de una hora con velocidad menor a $0,6 \mathrm{Kn}(\mathrm{Kn}=$ nudos) (Figura 3). No se computaron buques en navegación (velocidad sostenida $>$ 0,6 Kn durante más de una hora). De esta forma se identificaron las zonas de fondeo/espera/alijo, discriminando el número y tipo de buques presente en cada una, el tiempo de residencia y su puerto o zona de destino.
Figura 2. Distribución del esfuerzo de pesca de flota costera uruguaya entre los años 2006 y 2015 (en rojo las zonas de mayor intensidad) y las zonas de alijo y fondeo. Fuente: modificado de Chocca et al., 2016. 
La localización de los buques en el área se realizó a través de AIS (Automatic Identification System) accediendo a la información de portales web públicos. El sistema se basa en que cada buque lleva a bordo un receptor-emisor que transmite en forma continua en banda de radio VHF la identificación del buque, coordenadas, rumbo, velocidad y destino, entre otros datos. El sistema es requerido para buques mayores de 300 GT (Gross Tonnage, GT, indicador de tamaño del buque, sin unidades) (SOLAS, 2002), y la información la reciben los buques en los alrededores y estaciones fijas en tierra generando una red que permite visualizar los movimientos de las unidades en el área para mejorar las condiciones de seguridad en la navegación. Los datos se presentan en sitios web de acceso público y comerciales agregando prestaciones (rastreo de buques, estado del tiempo, pronósticos) y alcance. El radio de localización basado en VHF alcanza $50 \mathrm{mn}$ dependiendo de las condiciones de transmisión por lo cual algunos sitios la complementan con receptores satelitales obteniendo localizaciones en tiempo real en áreas oceánicas, alejadas de las estaciones en tierra. Si bien la localización es muy precisa y continua, en ocasiones la señal se pierde por lo cual se utilizaron los datos presentados por varios proveedores simultáneamente para confirmar las posiciones (Marine Traffic, 2017; Vessel Finder, 2017; FleetMon, 2017).

El relevamiento se realizó entre agosto 2017 y febrero 2018 en dos modalidades: conteo diario de los buques fondeados y traslado de la información a un sistema de información geográfico (QGIS Development Team, 2017); y seguimiento individual de cada buque fondeado en cada zona anotando la posición del buque, fecha y hora de entrada y salida al área de fondeo, tipo de buque y destino (Cuadro 2).

Con la información colectada se calculó la ocupación de las zonas de fondeo (agosto 2017 a febrero 2018), y con el seguimiento individual la composición por tipo de buque, destinos y tiempo de residencia (diciembre 2017 a febrero 2018). Este seguimiento individual de ingreso, residencia y egreso del área se realizó en forma continua durante una semana cada mes. Los tipos de buque se agruparon en 10 grupos en función de su finalidad de uso y material transportado independientemente de sus dimensiones (Cuadro 3). De cada buque se anotó también la eslora total, tonelaje de porte muerto (Deadweight tonnage, DWT, capacidad de carga completa en toneladas métricas) y tonelaje bruto (OMI, 1969).

Cuadro 2. Resumen del conteo de embarcaciones en todas las zonas de fondeo (días observados) y del seguimiento individual (número de buques) entre diciembre 2017 y febrero 2018.

\begin{tabular}{|l|c|c|c|c|c|c|}
\hline Mes/año & $\mathbf{0 9 / 2 0 1 7}$ & $\mathbf{1 0 / 2 0 1 7}$ & $\mathbf{1 1 / 2 0 1 7}$ & $\mathbf{1 2 / 2 0 1 7}$ & $\mathbf{0 1 / 2 0 1 8}$ & $\mathbf{0 2 / 2 0 1 8}$ \\
\hline Días observados & 7 & 20 & 16 & 14 & 31 & $\mathbf{2 8}$ \\
\hline Seguimiento individual & & & & 101 & 108 & 96 \\
\hline
\end{tabular}




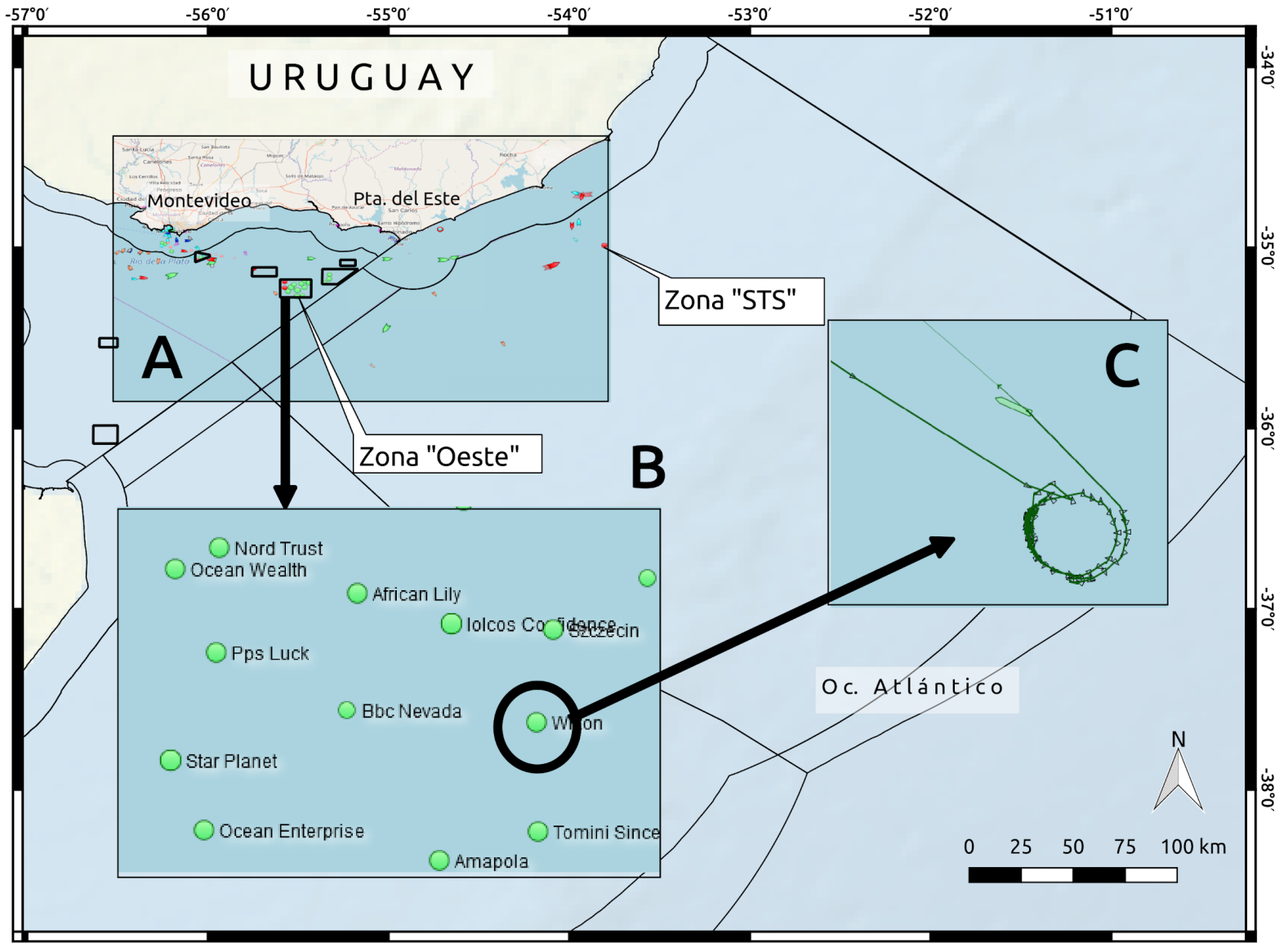

Cuadro 3. Denominación de tipos de buque resultantes de agrupar las clases de transporte identificadas en el área de estudio. Fuente: elaborado en base a datos de Marine Traffic (2017).

\begin{tabular}{|l|l|}
\hline Tipo & Transporte \\
\hline Granel & “Bulk carrier” transporte de cargas secas a granel \\
\hline Contenedores & Transporte de carga en contenedores estandarizados \\
\hline Carga general & Carga diversa \\
\hline Petróleo crudo & “Crude oil tanker” Buques cisterna de petróleo \\
\hline Productos químicos & “Oil/Chemical tanker”, Productos químicos a granel \\
\hline Ganado & “Livestock carrrier” Transporte de ganado en pie \\
\hline Vehículos & "Vehicules carrier” Transporte de carga rodada \\
\hline Frigorífico & “Reefer”, cargas refrigeradas \\
\hline Derivados de petróleo & “Oil / products tanker” Cisterna de productos derivados de petróleo \\
\hline Otros & $\begin{array}{l}\text { Científicos, remolcadores oceánicos, gas licuado, cisternas sin } \\
\text { especificar, etc. }\end{array}$ \\
\hline
\end{tabular}

El tiempo de residencia fue considerado entre la última emisión a velocidad mayor a 0,6 Kn en dirección a la zona de fondeo, y la primera emisión a dicha velocidad al abandonar la zona cuando se registraron consecutivamente durante más de una hora (Figura 2).

El puerto de destino considerado fue aquel indicado por el AIS y agrupados en base a la menor distancia entre ellos. Esta clasificación fue funcional a los efectos de simplificar la denominación en aquellos lugares donde se concentran puertos y terminales. Como
Figura 3. Vista general de la localización de los buques en la zona en los sitios web de AIS (A), detalle de la ubicación de cada buque (B), y posición de fondeo (C). La trayectoria circular en $\mathrm{C}$ corresponde a movimientos alrededor del ancla por efecto de corrientes y viento. Fuente: elaborado en base a datos de Marine Traffic (2017). 


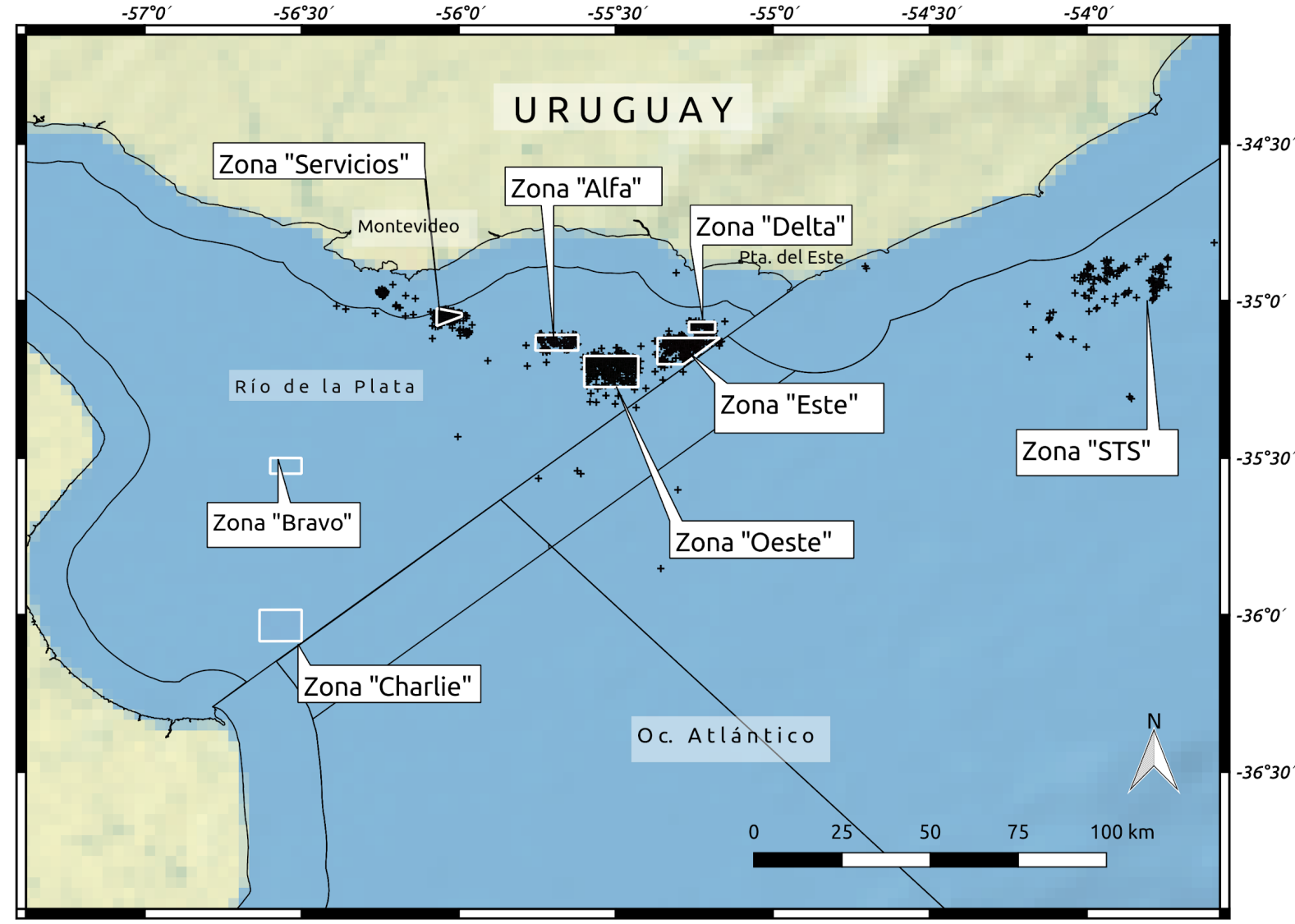

ejemplo, en algunas publicaciones se denomina "Gran Rosario" al conglomerado urbano sobre el Río Paraná que incluye desde "Timbúes" $45 \mathrm{~km}$ al norte, Puerto Gral. San Martín, San Lorenzo, Rosario, hasta "Villa Constitución" al sur; mientras que Massin (2015) identifica nodos más precisos a lo largo del Río Paraná.

\section{Resultados}

\section{Uso y ocupación de las zonas de fondeo}

La Figura 4 muestra el resumen de las localizaciones de buques fondeados para todo el período. Las concentraciones se ubicaron en todas las zonas del sector norte del RDP ("Alfa", "Servicios", "Este", "Oeste”, "Delta" y "STS"). Las concentraciones mayores se registraron en las zonas "Oeste", "Este" y "Servicios". En la zona "Charlie" fueron registrados menos de 5 buques, uno de ellos durante 30 días, y ninguno en la zona de alijo "Bravo". Algunos buques fueron registrados en las cercanías del canal de acceso a Montevideo, al Oeste en el $\mathrm{km} \mathrm{3}$, aunque no fueron considerados debido a que se encuentran dentro de las 7 millas de jurisdicción exclusiva de Uruguay.

A diferencia de las restantes zonas, los buques que operaron en la zona de transferencia STS mostraron un comportamiento más irregular, alternando períodos de fondeo con navegaciones a velocidad muy baja correspondientes a las operaciones de transferencia. La reglamentación define puntos de espera pero no una zona de fondeo poligonal como en las restantes.
Figura 4. Distribución acumulada de los buques fondeados en las diferentes zonas durante el muestreo diciembre 2017-enero 2018. Fuente: elaborado en base a datos de normativa y Marine Traffic (2017). 


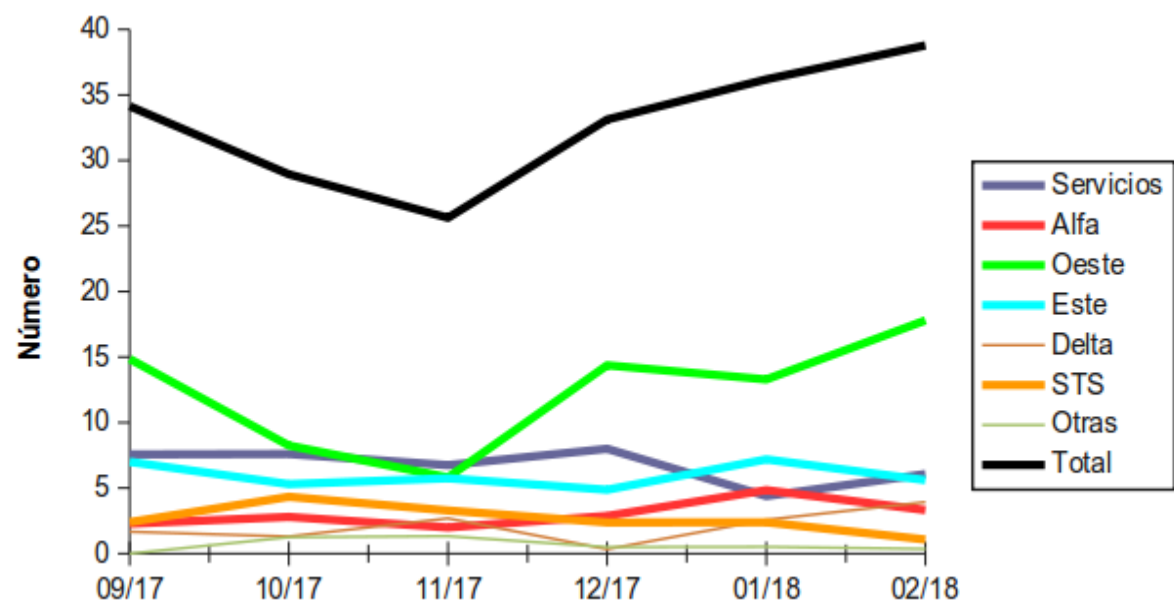

Un número bajo de unidades se encontraron fondeadas fuera de cualesquiera de las zonas, tanto en el RDP como en O. Atlántico, sin un patrón definido, pero en todos los casos en el sector norte del RDP.

El Cuadro 4 resume el número de buques fondeados en cada una de las zonas, encontrándose un promedio de 33 unidades diarias en toda el área, con un mínimo de 21 y un máximo de 48. Las zonas con mayor número de unidades fueron "Servicios" y "Oeste" con un promedio de 13 unidades por día.
Figura 5. Evolución del promedio de barcos ocupando las zonas de fondeo en el período de estudio. Fuente: elaborado en base a datos de Vessel Finder (2017), FleetMon (2017) y Marine Traffic (2017).

Cuadro 4. Número medio de embarcaciones fondeadas diariamente en las diferentes zonas entre setiembre 2017 y febrero 2018. Fuente: elaborado en base a datos obtenidos durante este trabajo.

\begin{tabular}{|l|c|c|c|c|c|c|c|c|c|}
\hline Mes & Servicios & Alfa & Oeste & Este & Delta & STS & C & Otras & $\begin{array}{c}\text { Total } \\
\text { general }\end{array}$ \\
\hline sep-17 & 7,6 & 2,3 & 14,9 & 7,0 & 1,7 & 2,4 & 0,0 & -- & 34,7 \\
\hline oct-17 & 7,6 & 2,8 & 8,3 & 5,3 & 1,3 & 4,3 & 0,0 & 1,3 & 29,7 \\
\hline nov-17 & 6,8 & 2,0 & 5,8 & 5,8 & 2,7 & 3,3 & 0,0 & 1,3 & 27,4 \\
\hline dic-17 & 8,0 & 2,9 & 14,4 & 4,9 & 0,3 & 2,4 & 0,0 & 0,5 & 33,5 \\
\hline ene-18 & 4,4 & 4,8 & 13,3 & 7,2 & 2,6 & 2,4 & 1,0 & 0,5 & 37,3 \\
\hline feb-18 & 5,4 & 4,2 & 12,4 & 1,6 & 5,4 & 1,3 & 0,8 & 0,4 & 33,0 \\
\hline
\end{tabular}

La Figura 5 muestra que el promedio mensual fue menor en noviembre incrementándose en enero y febrero 2018.

\section{Tiempo de residencia}

El tiempo de residencia promedio de los buques fue de 142 horas (5,9 días), con un mínimo de 1 hora y un máximo de 724 horas (30,2 días). Sin embargo la distribución de los tiempos de residencia fue fuertemente asimétrica, con mínimo $=0$, sin valores negativos y frecuentes valores extremos por lo cual la mediana resulta en un valor descriptivo más adecuado: 3,7 días (Figura 6).

El Cuadro 5 indica el tiempo de residencia (días) de los buques por zonas durante el período diciembre 2017-enero 2018. 


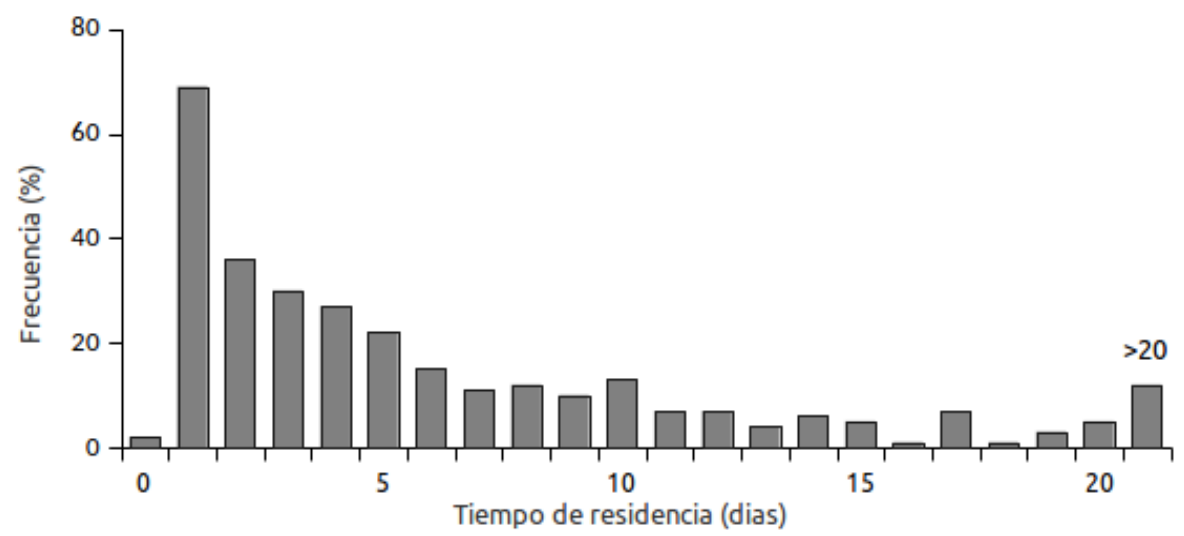

Cuadro 5. Tiempo de residencia en cada una de las zonas, rango (mínimo-máximo) y mediana de la distribución (días). * un solo buque que se desplazó a remolque. Fuente: elaborado en base a datos obtenidos durante este trabajo.

\begin{tabular}{|l|c|c|}
\hline Zona & Rango & Tiempo (días) \\
\hline Charlie & $28,1^{*}$ & $28,1^{*}$ \\
\hline STS & $1,3-24,7$ & 4,2 \\
\hline Este & $0,1-18,8$ & 7,3 \\
\hline Delta & $0,2-27,8$ & 6,4 \\
\hline Oeste & $0,1-28,2$ & 4,5 \\
\hline Alfa & $0,3-29,4$ & 2,8 \\
\hline Servicios & $0,1-30,2$ & 0,9 \\
\hline General & $0,1-30,2$ & 3,7 \\
\hline
\end{tabular}

\section{Tipos de buques}

El tipo de buque registrado con mayor frecuencia fue el granelero (Bulk carrier) seguido de los cisternas de productos químicos, de petróleo crudo, buques de carga general, transportes de contenedores, de derivados de petróleo y otros tipos diversos en menor frecuencia (Cuadro 6, Figura 7). No fueron encontrados buques militares ni de pasajeros. Transporte de petróleo crudo y portacontenedores fueron los de mayor eslora y tonelaje de registro, seguidos de buques de transporte a granel.

Cuadro 6. Composición de los tipos de buques para el período (diciembre 2017-febrero 2018), eslora promedio en $m$, y tonelajes (GT y DWT). Fuente: elaborado en base a datos obtenidos durante este trabajo.

\begin{tabular}{|l|c|c|c|c|}
\hline Tipo & $\%$ & Eslora $(\mathrm{m})$ & GT & DWT (t) \\
\hline Granel & 49,2 & 197 & 31614 & 55012 \\
\hline Productos químicos & 11,1 & 166 & 21108 & 34439 \\
\hline Petróleo crudo & 8,2 & 283 & 96168 & 179554 \\
\hline Carga general & 6,9 & 152 & 19254 & 29190 \\
\hline Contenedores & 7,2 & 274 & 75075 & 85372 \\
\hline Derivados petróleo & 5,2 & 180 & 27752 & 46576 \\
\hline Frigorífico & 2,6 & 139 & 8061 & 8630 \\
\hline Vehículos & 2,0 & 196 & 60211 & 19378 \\
\hline Ganado & 1,6 & 143 & 18079 & 9593 \\
\hline Otros & 5,9 & 122 & 20101 & 32294 \\
\hline
\end{tabular}




\section{Tipos de buques (\%)}

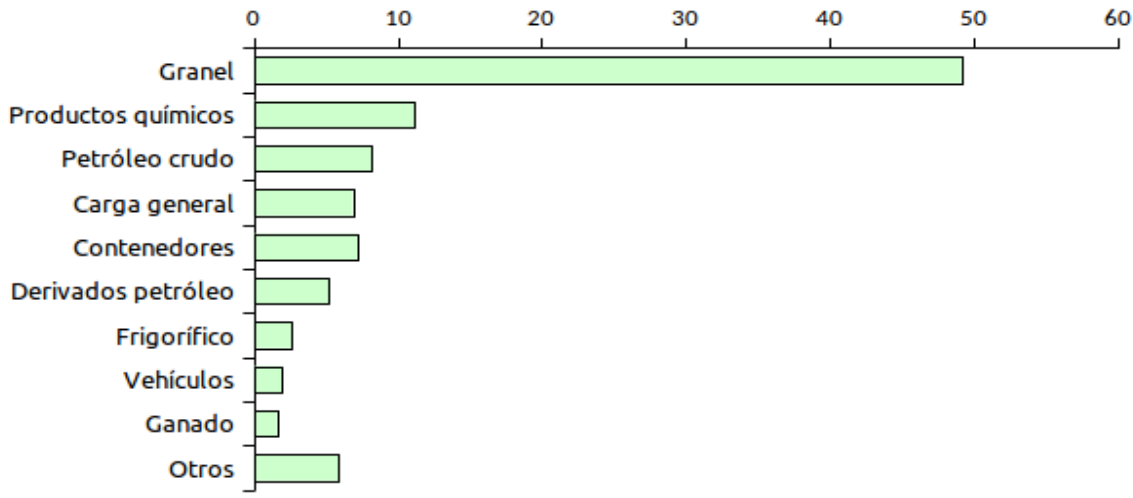

Tipos de buques porzona

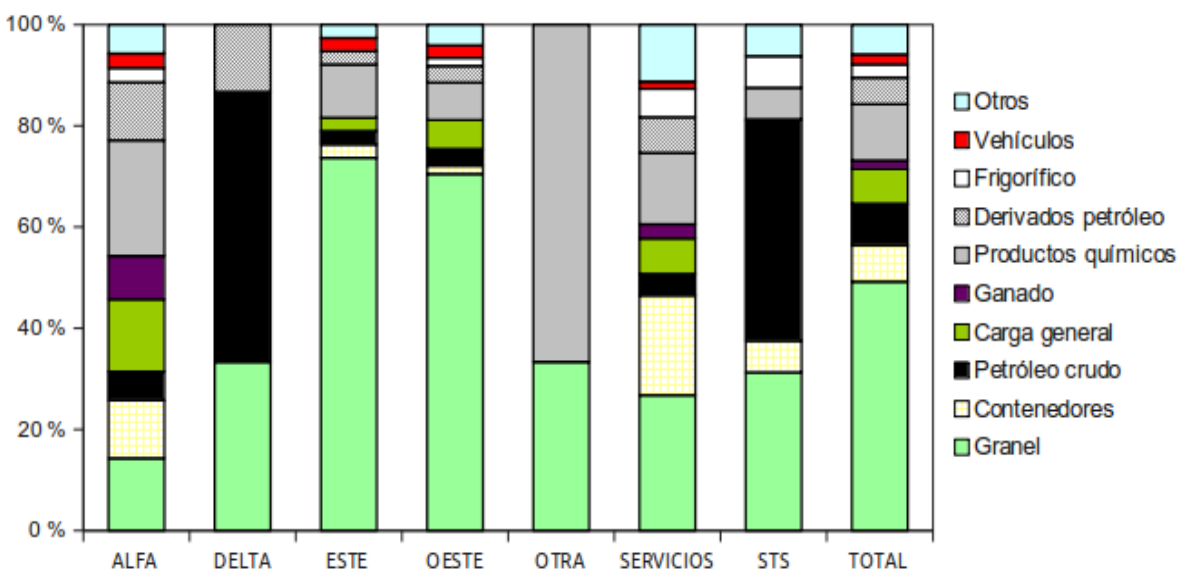

Los graneleros predominaron también en las zonas de espera Este y Oeste, transportes de petróleo crudo en las zonas STS y Delta, mientras que la zona de Servicios y Alfa mostraron la mayor diversidad de tipos de buques (Figura 8).

\section{Destinos}

Los destinos declarados incluyeron 37 puertos y terminales diferentes, y en varios casos zonas sin especificar: "Recalada", "Recalada Argentina", "Argentina", "High sea" o "USA".

La Figura 9 indica el país de destino de los buques en las zonas de fondeo, predominando los puertos de Argentina (59,7\%), de Uruguay $(25,1 \%)$ y puertos no identificados pero en el Río de la Plata (6,6\%, zona "Alfa” o "Recalada” sin especificar).

De los 303 barcos que indicaron destino, 26 fueron hacia puertos localizados en Brasil, Asia, África o aguas internacionales sin especificar. La mayor proporción (277 buques) se encontró en espera hacia puertos interiores y muy pocos hacia el exterior del RDP.

El Cuadro 7 resume los principales puertos de destino de cada país. En Argentina se destacan los puertos y terminales de San Lorenzo (San Lorenzo, Rosario, terminales Vicentín y San Benito, puerto Gral. San Martín), Buenos Aires (incluyendo La Plata), Campana (Campana, Zárate, Terminal Del Guazú y Las Palmas) y San Nicolás (San Nicolás de los Arroyos, Villa Constitución y Ramallo). En Uruguay los principales
Figura 7. Proporción de los diferentes tipos de buques en el período diciembre 2017-febrero 2018.

Fuente: elaborado en base a datos obtenidos durante este trabajo.
Figura 8. Composición de los tipos de buques registrados en cada zona del RDP en el período de estudio (excluidas zonas "Charlie" $y$ "Bravo"). Fuente: elaborado en base a datos de Marine Traffic (2017). 


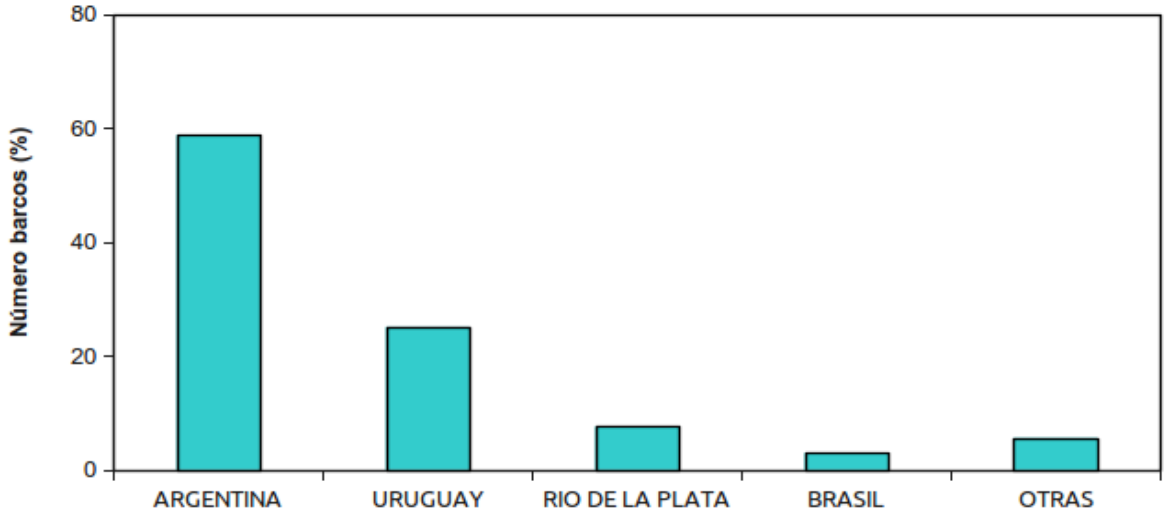

destinos indicados fueron Montevideo, La Paloma, Punta Pereira y Punta del Este (incluyendo Piriápolis y la Terminal de José Ignacio).

Cuadro 7. Resumen de los puertos de destino agrupados en zonas para cada país, en número de buques y porcentaje (diciembre 2017 - enero 2018). Fuente: elaborado en base a datos obtenidos durante este trabajo.

\begin{tabular}{|l|l|c|c|}
\hline País / Región & Puerto & Número & \% \\
\hline \multirow{4}{*}{ Argentina } & San Lorenzo & 104 & 37,5 \\
\cline { 2 - 4 } & Buenos Aires & 41 & 14,8 \\
\cline { 2 - 4 } & Campana & 18 & 6,5 \\
\cline { 2 - 4 } & San Nicolás & 12 & 4,3 \\
\cline { 2 - 4 } & Argentina Atlántico & 5 & 1,8 \\
\hline Río de la Plata & RDP & 20 & 7,2 \\
\hline Uruguay & Montevideo & 58 & 20,9 \\
\cline { 2 - 4 } & La Paloma & 7 & 4,0 \\
\cline { 2 - 4 } & Punta Pereira & 1 & 2,5 \\
\cline { 2 - 4 } & Nueva Palmira & & 0,4 \\
\hline
\end{tabular}

Si se excluyen buques cuyo destino fueron aguas exteriores al RDP (Brasil, China y otros países), puertos de Uruguay y Argentina sobre el O. Atlántico (Bahía Blanca, Santa Cruz y La Paloma), e incluso aquellos con destino no especificado en el RDP ("Recalada" y "zona alfa"), el 85\% de los destinos declarados correspondieron a buques que ingresaron a puertos interiores de Argentina (175 buques) y Uruguay (66 buques); y el 15\% a buques que permanecieron en zonas de fondeo o egresaron del RDP (Figura 10). La Figura 10 representa los destinos de los buques que se encontraron en las zonas de fondeo, indicando los buques que se dirigen a San Lorenzo, San Nicolás, Campana y Buenos Aires y luego egresan del RDP hacia el O. Atlántico. Las flechas rojas muestran la menor cantidad de buques hacia Montevideo y Nueva Palmira, y las flechas negras el ingreso y egreso de buques en operaciones de descarga en la zona de Pta. del Este o STS

Entre aquellos con destino puertos de Argentina, más del 50 \% se dirigían a San Lorenzo, seguidos de Buenos Aires, Campana y San Nicolás. La mayor proporción de buques que ingresan al RDP permite caracterizar las zonas de espera y fondeo como parte del viaje de entrada y en menos ocasiones como parte del viaje de salida.
Figura 9. País de destino de los buques fondeados en todas las áreas del RDP (diciembre 2017-enero 2018). Fuente: elaborado en base a datos obtenidos durante este trabajo. 


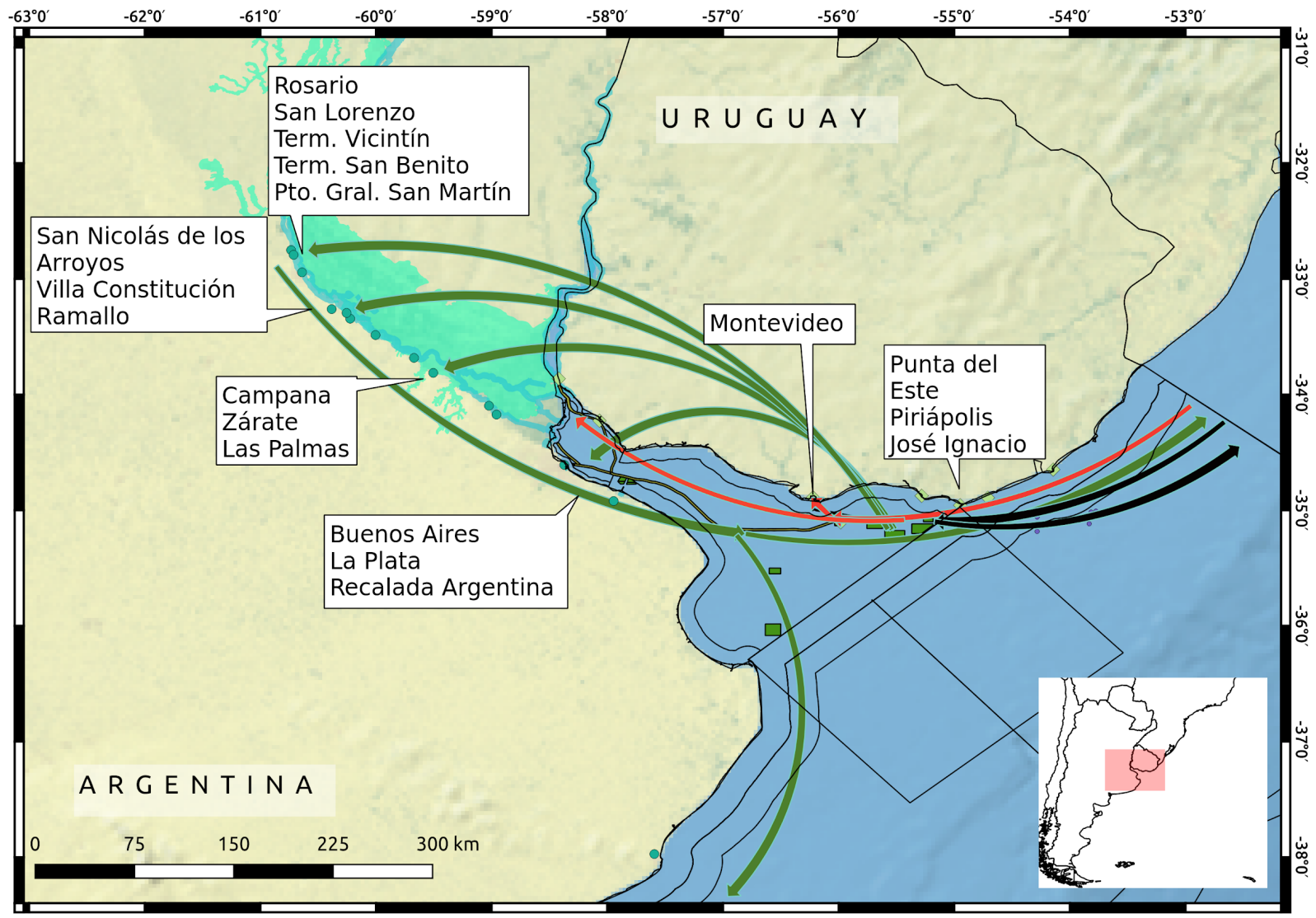

\section{Discusión y conclusiones}

La actividad en las zonas de espera y fondeo es continua, dinámica, y concentrada en el sector norte del RDP, más cercano a la costa uruguaya. Las zonas de alijo en el sector de Argentina ("Bravo" y "Charlie") no fueron utilizadas, mientras que aquellas del sector de Uruguay han sido aumentadas frecuentemente, estando las últimas incorporadas ("Oeste" y "Este") permanentemente ocupadas.

El número promedio de buques fondeados día a día fue de 33, oscilando entre 21 y 48 , y el tiempo de residencia medio de los buques de 3,6 días muestra un ingreso y egreso permanente de buques manteniendo la ocupación de las zonas. Comparados con datos provistos por una empresa proveedora del servicio AIS (Marine Traffic, 2017) el tiempo de residencia de los buques fue menor en 2013, con la mediana anual comprendida entre 0,45 días (zona "Oeste") y 3,08 días (zona "Este").

Es probable que el número oscile a lo largo del año debido a las variaciones del volumen de producción, almacenamiento y carga, estrategias comerciales, o por condiciones particulares. Como ejemplo, durante diciembre 2017 la prensa local informó de eventos que dificultaron las operaciones de carga de los buques en la región de San Lorenzo: una explosión en una terminal y medidas gremiales del transporte en febrero 2018. Estas condiciones coincidieron con un aumento del número de buques en la zona Oeste. En años anteriores se ha informado de situaciones de buques varados en el Río Paraná, o de bajo caudal, que impiden la navegación fluida y aumentaron el tiempo de espera (Bordón, 2012; ON24, 2014; Reuters, 2015).
Figura 10. Representación simplificada del destino de los buques. Las flechas verdes indican los buques que se dirigen a San Lorenzo, San Nicolás, Campana y Buenos Aires y luego egresan del RDP hacia el O. Atlántico. Las flechas rojas muestran los buques hacia Montevideo y Nueva Palmira, y las flechas negras el ingreso y egreso en operaciones de descarga en Punta del Este o STS. Fuente: elaborado en base a datos obtenidos durante este trabajo. 
La mayor parte de las zonas, y en particular las de mayor extensión (zonas "Este" y "Oeste") fueron ocupadas por buques que se dirigen a puertos de Argentina, en particular Rosario y San Lorenzo. Esto las convierte en una zona secundaria de operaciones para movimientos de cargas de Argentina y resulta en un uso de los espacios del RDP fuertemente asimétrico. Esta asimetría repercute directamente sobre el uso de la zona para operaciones pesqueras, en particular de Uruguay, debido a la cercanía del puerto de operaciones (Montevideo), y a que ocupa una zona activa de pesca (Chocca et al. 2007; Chocca et al. 2016; Marín et al. 2007, 2013).

Si bien en estas zonas no se prohíbe la pesca, de hecho resulta poco practicable con redes de arrastre de fondo, el arte de pesca usado por la totalidad de la flota costera nacional. La operación con una red que opera sobre el fondo en zonas donde se encuentran anclas y cadenas de los buques fondeados resulta en un peligro evidente. Las dimensiones de los graneleros fondeados en la zona Oeste requieren mantener un espacio amplio entre buques para evitar colisiones, que se observó fue cercana a $3 \mathrm{~km}$. Buques de estas características (190 m de eslora y 55000 DWT) requieren un radio de giro de unos 800 $\mathrm{m}$, anclas de 7 a $9 \mathrm{t}$ de peso además de la cadena (aproximadamente $166 \mathrm{~kg} / \mathrm{m}$ ), y una distancia de seguridad no menor a 3 veces la profundidad más al menos una eslora.

$\mathrm{Al}$ variar el número de buques varía la superficie ocupada. Se observaron pesqueros alrededor de las zonas de espera, entre las zonas de espera, y excepcionalmente entre barcos fondeados a velocidades bajas aunque no es claro si efectivamente se encontraban en operaciones de pesca. En ese sentido, estas áreas se convierten en una zona de restricción a la pesca con arrastre que se agrega a las zonas de veda basadas en motivos biológicos y otras, y debería ser considerada en la estimación del área "pescable". La disminución del área "pescable" tuvo efectos en las medidas de conservación y ordenación adoptadas por Uruguay entre 2007 y 2010 cuando fue aumentada la zona de prohibición de operaciones con red de arrastre ampliando la franja de 5 a $7 \mathrm{mn}$ sobre la costa entre la Isla de Flores y el límite lateral con Brasil. Esta medida fue rechazada por las agrupaciones de pescadores y anulada tras una sentencia del Poder Judicial en 2011 (Sentencia Tribunal de lo Contencioso Administrativo, TCA N²07, 15 marzo 2011).

El uso asimétrico de los espacios tiene consecuencias negativas para Uruguay en varios aspectos. El Tratado del Río de la Plata garantiza la libertad de navegación, lo cual se cumple en todos los casos. Sin embargo la situación es diferente con el uso de las zonas de fondeo y espera, ya que el número de buques en el área ha cambiado desde la concepción inicial de delimitación de áreas similares cercanas a las costas de Uruguay y Argentina de 1973.

Un alto porcentaje de los buques fondeados indicó como destino San Lorenzo, que junto con Rosario y zonas cercanas constituyen un nodo importante en la producción y exportación de cereales de la región pampeana de Argentina. Se estima que en 2016 ingresaron 2180 buques al Gran Rosario, un 75 \% del total nacional (Bergero et al., 2016). En dicho año la región pampeana lideró las ventas al exterior (74\% de las exportaciones totales de Argentina), con una fuerte incidencia de manufacturas de origen agropecuario, manufacturas de origen industrial y productos primarios (INDEC, 2016).

En el mismo año Argentina despachó al exterior aproximadamente 87,8 millones de toneladas de granos, harinas y aceites, con el Gran Rosario como nodo portuario principal que habría remitido al exterior cerca de 67,7 millones de t (77\% de las exportaciones totales) de productos nacionales y mercadería de origen paraguayo y boliviano que llega en barcazas por el Río Paraná (Bergero et al., 2017). Rossi y Terré (2017) indican también que en la zona de Rosario se origina el $57 \%$ de la producción nacional de soja, el $47 \%$ de la de maíz y el $41 \%$ de la de trigo, y la distribución de los embarques de granos sigue un patrón 
estacional muy correlacionado al de su producción con la mayor parte de los embarques en el período de cosecha. Según estos autores la mayor parte de los embarques de maíz se realiza entre marzo y julio; de soja entre abril y agosto, y los de trigo entre diciembre y abril del año siguiente. Los meses con mayor frecuencia de embarques coinciden con la mayor captura de corvina por la flota uruguaya en el área del Tratado, que se produce entre mayo y octubre (CTMFM, datos estadísticos 2009-2014).

Partiendo de una producción de granos, harinas y aceites de 54,7 millones de toneladas en 2012/2013, Bergero y Calzada (2016) estiman que podrían alcanzarse 65,6 millones de toneladas en $2019 / 2020$, y que el tránsito de buques podría modificarse si se realizan obras de dragado que permitan utilizar buques mayores y descongestionar las vías navegables.

En todos los casos, el número de buques en espera en el Río de la Plata tiene influencia en el espacio físico para las operaciones pesqueras, disminuyéndolo y concentrándolo en las zonas de mayor profundidad del sector norte cercano a la costa de Uruguay. En un escenario de crecimiento de la producción agrícola regional como el descrito, es de esperar un aumento de embarques y por lo tanto de buques en número y tamaño.

En ese sentido deberían manejarse alternativas que disminuyan o compensen este efecto que pueden abarcar desde la aplicación de tarifas por uso del área hasta el rediseño de las zonas de alijo, fondeo y espera que cambien el balance del uso espacial.

NOTA: Las opiniones vertidas en este trabajo son de exclusiva responsabilidad de los autores y no comprometen a las Instituciones en las que trabajan.

\section{Agradecimientos}

Un especial agradecimiento a Miluše Tichavska (Marine Traffic) que proporcionó información que hizo posible comparar los datos obtenidos en este trabajo con antecedentes en años previos de la zona. 


\section{Q Bibliografía}

» Barattini, L. P. (1943). Problemas de la pesca y fluctuación del pescado en Uruguay. Rev. Med. Vet. Y Paras, VII (1 al 4).

»Bergero, P., Calzada, J. (2016). Despachos por el Paraná desde Rosario pasan de 55 a 73 Mt. Bolsa de Comercio de Rosario. Informativo semanal, XXXIV (1770).

»Bergero, P. Calzada, J., Sesé, A. (2017). Estimación de la logística del transporte granelero en el país. Bolsa de Comercio de Rosario. Dirección de Informaciones y Estudios Económicos, XXXV (1808).

» Bordón, J. (2012). Un barco encallado paraliza los puertos del Gran Rosario. En La Nación, 22 enero 2012.

" https://www.americaeconomia.com/negocios-industrias/autoridades-liberanbuque-varado-en-principal-zona-agroportuaria-de-argentina

»Cámara De Representantes (2015). Intergremial marítima. Parlamento, Uruguay. XLVIII Legislatura. Comisión de Legislación del Trabajo, 70, <http://www. diputados.gub.uy/wp-content/uploads/2015/06/VT-0070.pdf>.

»CARP (2017). El Río de la Plata. Comisión Administradora del Río de la Plata (CARP). <http://www.comisionriodelaplata.org/el_rio.asp>.

»Chocca, J., González, B., Marín, Y., Beathyate, G. (2007). Resumen del área de operación de la flota pesquera uruguaya a través del sistema de información pesquera satelital (SIPESAT). Categoría "B". Enero 2005 - Marzo 2007. DINARA, Laboratorio de Tecnología Pesquera. Informe Técnico nํㅜ․ Mayo, 2007. 35 p.

»Chocca, J., Marín, Y., González, B., Beathyate, G., López, G. (2016). Actividad de la flota pesquera industrial uruguaya discriminada por categoría en el Río de la Plata y Zona Común de Pesca Argentino-Uruguaya entre los años 2006 y 2015. Laboratorio de Tecnología Pesquera, Dirección Nacional de Recursos Acuáticos DINARA. Informe interno, Octubre, 2016. 47 p.

»CTMFM (2017). Comisión Técnico Mixta del Frente Marítimo. http://ctmfm.org/

» CIC (2012). Comité Intergubernamental Coordinador de los Países de la Cuenca del Plata (CIC). Programa Marco para la Gestión Sostenible de los Recursos Hídricos de la Cuenca del Plata, en Relación con los Efectos de la Variabilidad y el Cambio Climático. <https://proyectoscic.org/lacuencadelplata/poblacion-yeconomia>.

»DIEA (2017). Anuario estadístico agropecuario 2017. Estadísticas agropecuarias. MGAP. 214 P.

»DINARA (2015). Boletín estadístico pesquero 2015. DINARA - MGAP. 60 p.

" DISMAR (2017). Disposición Marítima no 9 (1977); Disposición Marítima no 79 (2000); Disposición Marítima no 156 (2015); Disposición Marítima no 167 (2017). Disposición Marítima, Armada Nacional, Uruguay. <http://www.armada.mil. uy/Pagina/institucion/prena/dirme/disposiciones-maritimas.html>.

» FLEETMON (2017). Vessel position tracking. <https://www.fleetmon.com/>.

»Fossati M., Santoro, P., Mosquera, R., Martínez, C., Ghiardo, F., Ezzatti, P., Pedocchi, F., Piedra-Cueva, I. (2014). Dinámica de flujo, del campo salino y de los sedimentos finos en el Río de la Plata. En RIBAGUA - Revista Iberoamericana 
del Agua, Volume 1, Issue 1, 2014, 48-63, <https://doi.org/10.1016/S23863781(15)30007-4>. $\quad<$ http://www.sciencedirect.com/science/article/pii/ S2386378115300074>.

" González, B., Chocca, J., Marín, Y., Beathyate, G., Ortega, L. (2008). Distribución trimestral del esfuerzo de pesca de la flota costera uruguaya (categoría B) y su relación con la temperatura superficial del mar. DINARA, Laboratorio de Tecnología Pesquera, Laboratorio de Oceanografía. Informe Técnico nº $9.1^{\circ}$ de julio.

» INDEC (2016). Comercio exterior. Origen provincial de las exportaciones, año 2016. Instituto nacional de Estadística y Censos (INDEC). Ministerio de hacienda, Argentina. Informes Técnicos, 1 (37), 1 (5).

» Marine Traffic (2017). Global ship tracking intelligence, AIS marine traffic. <https:// www.marinetraffic.com/>.

» Marín, Y., Chocca, J., González, B., Beathyate, G. (2007). Resumen del área de operación de las unidades de pesca categoría B durante el año 2006 y su relación con la extensión de áreas de prohibición de uso de redes de arrastre de fondo. DINARA, División Técnica. Laboratorio de Tecnología Pesquera. Informe Técnico 4, DT/LTP/o04. Mayo, 2007.

» Marín, Y., Chocca, J., González, B., Beathyate, G. (2013). Interacciones entre la actividad pesquera y los proyectos de desarrollo en la Zona Económica Exclusiva uruguaya. Frente Marítimo, 23, 29-53.

» Massin, T. (2015). Dinámicas portuarias del litoral metropolitano del Paraná desde 1990. Revista Transporte y Territorio, 12, 48-69.

" Observador (2015). Sector pesquero local redujo a la mitad su producción en un año. El Observador, Economía y Empresas, 11 agosto 2014. <https://www. elobservador.com.uy/sector-pesquero-local-redujo-la-mitad-su-produccionun-ano-n285327>.

»ON24 (2014). Las consecuencias de los buques varados en el Paraná. En Cronista, 20 marzo 2014. <http://www.on24.com.ar/locales/las-consecuenciasde-los-buques-varados-en-el-paran/>.

» OMI (1969). International Convention on Tonnage Measurement of Ships. Adoption: 23 June 1969; Entry into force: 18 July 1982. OMI, Organización Marítima Internacional.

"QGIS Development Team (2017). QGIS Geographic Information System. Open Source Geospatial Foundation Project. <http://qgis.osgeo.org>.

»Reuters (2015). Buque varado demora 48 barcos en principal vía exportadora de granos de Argentina. Reuters, 20 mayo 2015. <http://www.agritotal.com/nota/ buque-varado-demora-48-barcos-en-principal-via-exportadora-de-granos-deargentina/>.

»Riguetti, B. (2016). La pesca en Uruguay atraviesa una profunda crisis. En Caras y caretas, 13 marzo 2016. <https://www.carasycaretas.com.uy/la-pesca-enuruguay-atraviesa-una-profunda-crisis/>.

»Rossi, G., Terré, E. (2017). 5 preguntas sobre patrones de logística en los granos que Argentina exporta. Bolsa de Comercio de Rosario. Informativo semanal. Dirección de Informaciones y Estudios Económicos. Año XXXIV - N $1766-7$ de julio 2016.

"Solas (2002). AIS - IMO Resolution A.917 (22) Automatic Identification Systems (AIS), Convenio internacional para la seguridad de la vida humana en el mar, 
1974 (Convenio SOLAS). SOLAS regulation V/19, paragraph 2.4. https://puc. overheid.nl/nsi/doc/PUC_1361_14/2/

»Vessel Finder (2017). Rastreo de buques AIS de tráfico marino gratuito. https:// www.vesselfinder.com/es

\section{Yamandú H. Marín / ymarin@cincytema.com.uy}

MSc Ciencias Ambientales, MSc Marine Spatial Planning, participa en el Laboratorio de Tecnología Pesquera (DINARA, Ministerio de ganadería, Agr. Y Pesca, Uruguay) y en grupos de investigación en Facultad de Ciencias en Montevideo, Uruguay (CINCYTEMA, UNDECIMAR). Este trabajo es parte del programa de doctorado (PEDECIBA, Uruguay).

\section{Guzmán López / guzilop@gmail.com}

Lic. en Ciencias Biológicas (Facultad de Ciencias en Montevideo), Desarrollador y Analista en Tecnologías de Información. Investigador del Laboratorio de Tecnología Pesquera (DINARA, Ministerio de ganadería, Agr. y Pesca, Uruguay).

\section{Julio F. Chocca / jchocca@dinara.gub.uy}

Patrón de pesca (UTU-CETP, Uruguay) e investigador del Laboratorio de Tecnología Pesquera (DINARA, Dirección Nacional Recursos Acuáticos, Ministerio de ganadería, Agr. y Pesca, Uruguay).

\section{Mónica Gómez / mgomezerache@gmail.com}

MSc en Cambio global, Docente e investigador de la Facultad de Ciencias (Montevideo, Uruguay), integrante del grupo de investigación CINCYTEMA, Ciencia y Tecnología Marina, Facultad de Ciencias, Universidad de la República, Uruguay. Este trabajo es parte del programa de doctorado (Universidad de Aveiro, Portugal). 